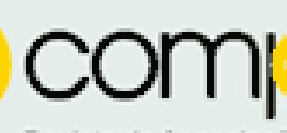

Revista da Associacajo Nacional

dos Programas de Pós-Graduaça

em Comunicacto

Este artigo foi publicado na edição 1, em dezembro de 2004, da revista eletrônica e-compós: http://www.compos.org.br/e-compos

\title{
INTERSEÇÕES DA TEORIA CRÍTICA CONTEMPORÂNEA: ESTUDOS CULTURAIS, PÓS-COLONIALISMO E COMUNICAÇÃO
}

\section{Angela Prysthon ${ }^{1}$ Universidade Federal de Pernambuco}

Já se vão mais de dez anos da publicação do livro $O$ local da cultura, de Homi K. Bhabha pela Routledge. E, para além do valor de efeméride, podemos ver na passagem desse período a consolidação de uma tendência nos Estudos Culturais e na teoria crítica: o pensamento pós-colonial e sua influência em campos tão diversos como a sociologia, ciência política, teoria literária, antropologia e comunicação. É evidente que outros autores são tão importantes (ou mais) que Bhabha nessa tendência (Stuart Hall, Edward Said, Gayatri Spivak, Arjun Appadurai, entre outros), mas o livro de Bhabha pode servir como emblema da infiltração dos problemas relacionados com a diferença cultural e a agência periférica nas Ciências Humanas como um todo. Assim, O local da cultura (e todos os conceitos que ele reuniu e reintroduziu - entrelugar, agência, negociação identitária, entre outros) funciona como uma espécie de desculpa, ou epígrafe estendida, para falar desse percurso dos Estudos Culturais ao longo desses dez anos.

\footnotetext{
${ }^{1}$ Professora do Programa de Pós-graduação em Comunicação da Universidade Federal de Pernambuco prysthon@uol.com.br
} 
Este artigo visa abordar as transformações da teoria crítica da cultura sobre e no Terceiro Mundo a partir da posição periférica, a partir das margens. Pretendese apresentar um breve panorama da "história (ou histórias) da teoria" das duas últimas décadas, especialmente no que se refere aos conceitos de identidade, minorias, agência, representação de nacionalidade na pós-modernidade. Partindo da própria noção de Terceiro Mundo, a intenção é analisar como algumas ramificações teóricas contemporâneas, como, por exemplo, as teorias da hibridez cultural e da tradução da diferença social, podem ir além das polaridades Ocidente/ Oriente, Mesmo/Outro, Centro/periferia.

A partir dos anos 80 surge um termo substituto (enquanto instância teórica e talvez até como instância política) para o Terceiro Mundo. Pós-colonial, póscolonialismo, substituem Terceiro Mundo nas esferas da produção acadêmica e polêmicas intelectuais. Não por acaso a terminologia pós-colonialista aparece num contexto onde aquela esperança de unidade terceiro-mundista, mais do que enfraquecida, está totalmente eclipsada por crises internas e externas no Terceiro Mundo. O que se evidencia a partir dos anos 80 é que não só que o Terceiro Mundo não é um bloco homogêneo (algo que sempre esteve implícito em todas as discussões relevantes sobre o Terceiro Mundo, desde a aparição do rótulo) como também que o Terceiro Mundo não pode e não quer se identificar consigo mesmo enquanto bloco homogêneo.

Outro tipo de atitude terceiro-mundista aparece, tanto no campo teórico, como no cultural: aquele que tenta usar a "diferença”, a "alteridade" como ponto de partida para a integração ao modelo capitalista global, especialmente em relação aos bens culturais. As indústrias culturais mundiais abrem-se ao chamado multiculturalismo e os efeitos de uma cada vez maior presença de bens simbólicos periféricos junto à cultura de massa internacional se fazem sentir em todos os cantos do planeta, especialmente desde o início da década de 80.

Assim, quando as alternativas culturais libertárias propostas pelo terceiro mundismo militante nos anos 60 e 70 são substituídas a partir dos anos 80 pelas estratégias de mercado transnacional, as abordagens teóricas sobre o Terceiro Mundo também têm que mudar. O que acontece com as teorias sobre o "Terceiro Mundo" nos 80 é uma transferência de campos do conhecimento: o que antes era o 
quase absoluto domínio das ciências políticas e sociais agora faz mais parte da história e mais especificamente da história da cultura, estudos culturais, midiáticos e literários.

O multiculturalismo (como inicialmente é chamada a disseminação de diversas culturas no Ocidente no final dos anos 80 e que pode ser denominado também de “estado híbrido", “mundialização”, "globalização cultural” (CANCLINI,1990, 1999; ORTIZ, 1994; FEATHERSTONE, 1995) vai ultrapassar as fronteiras de um mercado cultural de massas mais sofisticado e acaba por tomar conta também da academia- principalmente dos círculos anglo-americanos- como fenômeno pós-moderno (visto assim como conseqüência de um dos traços da pósmodernidade - a des-centralização). E assim como acontece com tudo relativo à pós-modernidade na primeira metade dos anos 80, todas as questões relacionadas com o multiculturalismo ocupam lugar de destaque nas principais discussões culturais na segunda metade desta década.

Por um lado, o debate sobre multiculturalismo tem resvalado desde então para uma oposição extrema entre conservadores e radicais multiculturalistas, acabando por isolar cada parte nas suas tentativas de provar a superioridade de sua cultura. Por outro, é reaceso o interesse cultural no Outro para além da psicologia, antropologia, lingüística e etnografia. O Outro que emerge no final dos anos 80 nos cursos universitários europeus e norte-americanos é sobretudo o “Terceiro Mundo” (claro, também a mulher, os gays e lésbicas, os negros, mas para os propósitos de delimitação do território pós-colonial é a reemergência da temática terceiromundista-com outros nomes- que vai ser importante aqui). E em especial assuntos concernentes às relações entre "Império" e "Colônias", ou "ex-colônias".

Daí o termo, pós-colonial. A partir do termo, uma série de antologias, cursos, tratados, ensaios, todos com relativo sucesso de marketing garantido, pelo menos nos Estados Unidos e Grã-Bretanha. As teorias culturais do final do século XX estão indissoluvelmente impregnadas por esse novo interesse pelo Outro, interesse que tem diversas naturezas (“científicas”, culturais, mercadológicas...) e onde todas podem conviver numa mesma teoriaCoro num mesn coloca Ella Shohat, 
the "post-colonial" did not emerge to fill an empty space in the language of political-cultural analysis. On the contrary, its wide adaptation during the late eighties was coincident with and dependent on the eclipse of an older paradigm, that of the Third World. (SHOHAT, 1992, 100) (O pós-colonial não emergiu para preencher um especo vazio na linguagem da análise políticocultural. Ao contrário, a sua larga adaptação durante o final dos anos oitenta foi coincidente com e dependente do eclipse de um paradigma anterior, aquele do Terceiro-Mundo.)

O paradigma "Terceiro Mundo" desaba porque é excessivamente indiferenciador, porque homogeniza diferenças e principalmente porque vem carregado com uma essência revolucionária advinda das lutas de independência nos anos 60 e 70. E a teoria pós-colonial que de certo modo o substitui não deixa de ser ela também homogeneizadora: entretanto, ao impor o colonialismo como algo "passado", inevitavelmente o componente utópico-revolucionário é suprimido desta proposta teórica. O que pode ser mais conveniente para as consciências acadêmicas primeiro-mundistas. Em contrapartida, destituir a idéia de Terceiro Mundo e substituí-la por periferia na linguagem coloquial e por pós-colonial no sentido teórico é também um movimento que parte de terceiro-mundistas que não se reconhecem como tal ou que se sentem diminuídos com esse tipo de classificação:

in India, people who can think of the three worlds explanation are totally pissed off by not being recognized as the centre of the nonaligned nations, rather than a "Third World country". (SPIVAK, 1990, 91) (Na Índia, as pessoas que podem pensar na explicação dos três mundos estão totalmente irritadas pelo país (a Índia) não ser reconhecido como o centro das nações não-alinhadas, ao invés de um “país de Terceiro Mundo”).

No sentido estritamente acadêmico, o surgimento da teoria pós-colonial vem tentar resolver certos problemas embutidos no "multiculturalismo radical". Ao fundir todas as "etnias e histórias" em um só rótulo (pós-colonial), os intelectuais pós-coloniais não se isolam tanto quanto, digamos, um professor de "Estudos africanos", mesmo que estes intelectuais estudem um mesmo fenômeno. Ao 
introduzir o contexto pós-colonial, estes teóricos estão assumindo que tal fenômeno tem que ser visto em relação a outras experiências e dados deste contexto. Ao mesmo tempo, também podem considerar países do Primeiro Mundo como pós-coloniais, já que agora não estão em jogo espaços geográficos e sim condições temporais. A teoria pós-colonial tenta, então, abarcar a cultura mundial depois que a experiência colonial “já passou”. Assim, tomando como "passada” tal experiência. O que parece inevitavelmente controverso quando a condição colonial, em vários casos, ainda persiste. Ademais, a teoria pós-colonial não considera um fator fundamental para a maioria dos países "terceiro-mundistas" ou “pós-coloniais": o neocolonialismo. Somemos a isso o fato de que há países "póscoloniais" que são "pós-coloniais" há mais de dois séculos - o que não apaga o seu passado colonial - e de que cada experiência colonial é uma experiência colonial diferente (há diversos tipos de colonizadores, colônia e colonizados), e temos para a teoria pós-colonial uma premissa de certo modo limitadora.

Outra limitação se refere ao idioma no qual essa teoria vem sendo majoritariamente divulgada. Não se pode esquecer que o conceito de pós-colonial foi se acomodando dentro dos limites de um território lingüístico determinado - o da língua inglesa. Mesmo que se isso não implique na negação de espaços a abordagens de outras experiências pós-coloniais, demarca o lugar especial que ocupam as ex-colônias (que só no século XX se tornaram ex-colônias, cabe lembrar ...) européias - especialmente britânicas - da Ásia, África e Caribe nas universidades anglo-americanas hoje. Se no conceito e na atitude do "Terceiro Mundo" espalhados nos anos 60 e 70 havia a predominância do anticolonialismo como um projeto único para todos os países terceiro-mundistas, no pós-colonial é como se ele não fosse mais necessário. O que tem se sobressaído por enquanto, como ponto de intersecção entre os adeptos do projeto pós-colonial é, nas suas instâncias mais básicas, a língua inglesa como signo de uma reação específica ao Império Britânico e da influência da diáspora que levou tantos intelectuais terceiro-mundistas à outra força hegemônica da língua inglesa, os Estados Unidos. E em segundo lugar, a capacidade que essa teoria tem de se tornar uma mercadoria tão facilmente negociável no circuito acadêmico anglo-americano (até por sua estreita ligação com outros “pós”: o pós-estruturalismo e o pós-modernismo). 
Uma das primeiras conseqüências da globalização é o enfraquecimento da noção de estado-nação, o que pode sugerir, de certa maneira, a gradual deshierarquização dos países europeus ou dos Estados Unidos como centros irradiadores de modismos culturais mundiais. Mas isso não significa que as grandes metrópoles mundiais percam seus postos de potências culturais. Nova York, Paris, Londres ainda são tanto algumas das peças mais fundamentais do imaginário metropolitano do Ocidente, como líderes incontestes de tendências artísticas e do mercado cultural mundial. Cidades como essas determinam a condição periférica como principal tendência das modas culturais de fim de milênio. Ou seja, o próprio centro desestabilizando a sua condição de centro com o multiculturalismo.

Os Estudos Culturais se estabelecem como o terreno por excelência tanto para o estudo como para o próprio desenrolar de transformações no âmbito de uma des-centralização cultural e das políticas internacionais da teoria. Os EC trazem à tona um ponto de vista muito mais abrangente - sendo simultaneamente bem específico na sua historicidade, fazem convergir um instrumental teórico que tenta revelar a contemporaneidade de maneira desmistificadora e des-hierarquizada e estabelecer uma política da diferença que desafie a hegemonia nordocêntrica, redefina a modernidade a partir de novos termos, aponte alternativas para um padrão cultural baseado na cópia e na imitação e garanta voz a sujeitos que anteriormente não tiveram direito a voz.

The new cultural politics of difference are neither simply oppositional in contesting the mainstream (or malestream) for inclusion, nor transgressive in the avant-gardist sense of shocking conventional bourgeois audiences. Rather, they are distinct articulations of talented (and usually privileged) contributors to culture who desire to align themselves with demoralized, demobilized, depoliticized, and disorganized people in order to empower and enable social action and, if possible, to enlist collective insurgency for the expansion of freedom, democracy and individuality. (WEST, 1994, 204) (As novas políticas culturais da diferença não são simplesmente oposicionais na sua 
contestação do mainstream (ou malestream) pela inclusão, nem transgressivas no sentido vanguardista de chocar o público burguês convencional. Ao contrário, elas são articulações distintas de colaboradores talentosos (e geralmente privilegiados) que desejam alinhar-se com pessoas desmoralizadas, desmobilizadas, despolitizadas e desorganizadas no sentido de empoderá-las e habilita-las para a ação social e, se possível, possibilitar uma insurgência coletiva pela expansão da liberdade, democracia e individualidade.)

São evidentes tentativas tanto dos Estudos Culturais, como da teoria póscolonial de revisar das desigualdades da modernidade e de apresentar alternativas teóricas aos modelos econômicos, sociais e políticos do "Primeiro Mundo". Neste contexto, a importância da revisão de um conceito como o de cosmopolitismo parece inegável inclusive por suas potencialidades de aplicação como uma terminologia muito mais precisa e aceitável que "relativismo cultural" ou “internacionalismo".

Alguns aspectos do contemporâneo têm que ser necessariamente levados em conta pelos Estudos Culturais e pela Teoria Crítica no que se refere ao cosmopolitismo: é explícita uma nova configuração urbana que torna caduca a noção da vivência da cidade como base do cosmopolitismo: algumas das maiores metrópoles do mundo não estão no centro, mas na periferia -Cidade do México, Jacarta, São Paulo, Istambul; a dissolução do chamado Segundo Mundo; a emergência dos países asiáticos como potências econômicas e as conseqüências culturais desse desenvolvimento econômico; o crescente isolamento cultural de alguns países muçulmanos; as guerras advindas de embates étnicos e religiosos; a diáspora dos intelectuais da periferia para o "Primeiro Mundo"; o avanço gigantesco das redes de comunicação: canais de tv a cabo, conglomerados da imprensa abrangendo vários países e, fundamentalmente como maior revolução, a Web, a Internet. Estes são alguns dos elementos que estabelecem novos parâmetros para as culturas periféricas e precipitam todas as dimensões culturais da globalização, parte do que Appadurai chama de "ampla modernidade” (2001). 
O pós-colonialismo, por exemplo, reafirma, como antes o terceiromundismo, mas agora de modo muito mais articulado teoricamente, o papel do periférico na História e a própria História periférica. A teoria pós-colonial é uma empresa de descolonização, mas não a descolonização concreta (algo que já foi mais ou menos realizado) das lutas armadas e acordos militares, mas a descolonização da História e da teoria, uma abordagem de fato alternativa do Ocidente. De teoria estritamente relacionada com as ex-colônias de língua inglesa a abordagem de muito maior escopo, os estudos pós-coloniais reinserem o debate da identidade nacional, da representação, da etnicidade, da diferença e da subalternidade no centro da história da cultura mundial contemporânea.

Comentando a obra de Gayatri Chakravorty Spivak, Robert Young considera a classificação de subalterno tanto para a historiografia produzida pelo "Outro", como o sujeito que a produz.

The subaltern historian not only locates historical instances of insurgency but also aligns him- or herself with the subaltern as a strategy for 'bringing hegemonic historiography to crisis' -which amounts to a good description of the strategic orientation of Spivak's own work. (YOUNG, 1990, 160) (O historiador subalterno (o subalternista) não apenas localiza instâncias históricas de insurgência, mas também se alinha à subalternidade como uma estratégia para "levar a historiografia hegemônica a uma crise" - o que resulta numa boa descrição da estatégia de orientação do próprio trabalho de Spivak. )

Ou seja, ao contrário da antropologia clássica ou da historiografia tradicional, a teoria pós-colonial poderia representar a periferia diretamente, mais do que isso -já que o pós-colonialismo contesta uma já ultrapassada concepção de representação-, é a própria voz do subalterno que está em jogo. A reescritura periférica da História, ou a desconstrução do Ocidente feita pelos estudos póscoloniais, portanto, implica num constante ataque à hegemonia ocidental e, se não uma completa inversão, uma reavaliação dos valores do cosmopolitismo convencional, uma reacomodação do cânone cultural, o des-centramento 
anunciado pelas teorias pós-modernas, enfim. O que não significa, contudo, que a revisão do cosmopolitismo implícita no pós-colonialismo seja a proposta de um relativismo cultural generalizado, a absolutização do relativismo. Como sugere Bruce Robbins, o cosmopolitismo pode ser uma denominação mais apropriada para a noção de “estado híbrido" e para a abrangência de certos aspectos do internacionalismo dos anos 60:

The interest of the term cosmopolitanism is located, then, not in its full theoretical extension, where it becomes a paranoid fantasy of ubiquity and omniscience, but rather (paradoxically) in its local applications, where the unrealizable ideal produces normative pressure against such alternatives as, say, the fashionable "hibridization". (ROBBINS, 1992, 183) (O interesse do termo cosmopolitismo está localizado, então, não na sua total extensão teórica, na qual se transforma numa fantasia paranóica de ubiqüidade e onisciência, mas, ao invés disso (paradoxalmente) nas suas aplicações locais, onde o ideal irrealizável produz uma pressão normativa contra alternativas como a tão na moda “hibridização".)

O cosmopolitismo pós-moderno, então, vai se constituindo como um cosmopolitismo quase que necessariamente periférico, tanto pelo problema da representação mencionado anteriormente, como pela óbvia e inerente experiência cosmopolita vivida no cotidiano da maioria das regiões periféricas. Embora isso se aplique à experiência do mundo urbanizado como um todo. Grandes metrópoles “nordocêntricas” como Nova York, Londres e Paris também têm no seu cotidiano uma experiência que inegavelmente se chama cosmopolitismo periférico. As zonas de contato entre "Primeiro" e "Terceiro" Mundos vão se multiplicando nas duas regiões e, como seria de se esperar, no destroçado "Segundo". A existência de bolsões de "Terceiro Mundo" no "Primeiro Mundo" e seu contrário, o "Primeiro Mundo" no "Terceiro Mundo" são não apenas a confirmação do cosmopolitismo periférico, como também uma condição sine qua non do capitalismo transnacional e o sinal de que um "mundo" somente está cada vez mais parecido na sua diversidade. Justamente no espaço intersticial, no fluido território intermediário, 
nessa zona de negociação entre "mundos", é que está localizado o arcabouço cultural que serve de objeto para a teoria pós-colonial e o instrumental teórico para analisá-lo.

Criticism formed in this process of the enunciation of discourses of domination occupies a space that is neither inside or outside the history of western domination but in a tangential relation to it. This is what Homi Bhabha calls an in-between, hybrid position of practice, or what Gayatri Chakravorty Spivak terms catachresis; "reversing, displacing, and seizing the apparatus of value-coding." (PRAKASH, 1992, 8) (A crítica formada nesse processo de enunciação de discursos de dominação ocupa um espaço que não está nem dentro nem fora da história do domínio ocidental, mas numa relação tangencial com ele. É o que Homi Bhabha chama de in-between, entrelugar, uma posição híbrida da prática teórica, ou o que Gayatri Chakravorty Spivak denomina catacrese; “a reversão, o deslocamento e a posse do aparato dos códigos valorativos").

O lugar do periférico na configuração da cultura contemporânea e na crítica, análise e teoria dessa cultura, portanto, está muito diferenciado em contraste com as disciplinas mais tradicionais. É um ponto de observação privilegiado no sentido da multiplicidade desse espaço intermediário. Mesmo que tantas outras teorias e estéticas já houvessem problematizado conceitos como representação, identidade, alteridade, hibridismo, colonização, Ocidente, Oriente; com o pós-colonialismo esses elementos são colocados num marco de referências que, ao invés de simplesmente inverter ou descartar termos e hierarquias, vai questioná-los na sua essência e na sua malha de inter-relações, vai pensar as condições de possibilidade, continuidade e de utilidade da sua construção.

Postcoloniality represents a response to a genuine need, the need to overcome the crisis of understanding produced by the inability of old categories to account for the world. (DIRLIK, 1994, 352) (A pós-colonialidade representa uma resposta a uma necessidade genuína: a necessidade de superar a crise de entendimento 
produzida pela inabilidade das velhas categorias em dar conta do mundo.)

No caso da América Latina, o pós-colonialismo vai estar irremediavelmente associado às teorias pós-modernas e ao discurso pós-estruturalista. Aí, a teoria pós-colonial vai desenvolver-se, sobretudo, como resposta às questões mais diretamente ligadas à modernidade e ao desenvolvimento social da região, além de ser um instrumental auxiliar relevante para os Estudos Culturais e para a Teoria da Comunicação. Neste contexto, sobressai-se especificamente a linha de trabalho sobre o hibridismo na cultura latino-americana. Hibridismo, híbrido, hibridização são conceitos-chave dos Estudos Culturais latino-americanos dos anos 90, prolongando discussões que haviam iniciado na abordagem do pós-moderno latino-americano na década anterior. Como havia sido dito antes, a obra de Néstor García Canclini ocupa um posto extremamente influente no debate sobre o estado híbrido da comunicação e da cultura latino-americana. Seu livro Culturas híbridas- Estrategias para entrar y salir de la modernidad postula a necessidade de uma abordagem "transdisciplinária” para a compreensão da cultura e da comunicação latino-americanas contemporâneas, estas fundamentalmente marcadas por uma intensa "heterogeneidade multitemporal". Canclini tenta ademais redefinir a modernidade - e tangencialmente a pós-modernidade - tendo em conta os processos de hibridização da América Latina.

Las reconversiones culturales que analizamos revelan que la modernidad no es sólo un espacio o un estado al que se entre o del que se emigre. Es una condición que nos envuelve, en las ciudades y en el campo, en las metrópolis y en los países subdesarollados. Con todas las contradicciones que existen entre modernismo y modernización, y precisamente por ellas, es una situación de tránsito interminable en la que nunca se clausura la incertitumbre de lo que significa ser moderno. (CANCLINI, 1990, 333)

Como no trabalho de outros latino-americanos e "latino-americanistas", a preocupação de Canclini com a modernidade (ou pós-modernidade) e seus processos especificamente latino-americanos o leva a elaborar outro trabalho onde 
a discussão da hibridização e mais ainda da emergência do multiculturalismo vão estar no cerne desses processos, nos quais é acentuada a tensão permanente entre o local e o global. A partir sobretudo de Consumidores y ciudadanos (1995), Canclini redimensiona principalmente a identidade urbana latino-americana moderna (ou pós-moderna) a partir das transformações do final do século. Seu interesse na nova configuração da cidade, principalmente na Cidade do México, mas na metrópole latino-americana de modo geral e suas indústrias culturais, desemboca na reflexão mais ampla sobre a pós-modernidade e a globalização.

As grandes cidades, dilaceradas pelo crescimento errático e por um multiculturalismo conflitante, são o cenário em que melhor se manifesta o declínio das metanarrativas históricas, das utopias que imaginaram um desenvolvimento humano ascendente e coeso através do tempo. Mesmo nas cidades carregadas de signos do passado, como a capital mexicana, o encolhimento do presente e a perplexidade diante do devir incontrolável reduzem as experiências temporais e privilegiam as conexões simultâneas no espaço. (CANCLINI, 1995, 130)

A vivência urbana - não a experiência da cidade moderna, mas toda a gama de complexidades e entrelaçamentos que surge de uma concepção pós-moderna de cidade - na América Latina passa a ser o foco central do pensamento de Canclini. Nas três conferências que constituem Imaginarios urbanos (1997), Canclini explicita ainda mais a inquietação sobre a pós-modernidade urbana na região, sempre retomando o debate acerca do contexto modernizador da globalização contemporânea e da hibridização cultural, ressaltando as particularidades dos processos de desterritorialização e reterritorialização latino-americanos e chamando a atenção para as possíveis interpretações equivocadas desses fenômenos - numa espécie de recado às teorias pós-coloniais:

Hay que aclarar en seguida que este reordenamiento global de las culturas no elimina las desigualdades ni la asimetría entre las metrópolis y las sociedades periféricas. Sin embargo, tampoco estamos en régimen de desigualdades comprensible con nociones de otro tiempo, como colonialismo o imperialismo. Es necesario 
construir una nueva conceptualización que vincule las desigualdades con las hibridaciones, de acuerdo con esta descentralización de los mercados globalizados, que ya no puede ser explicada, como hace Homi K. Bhabha, oponiendo la hibridación colonial a la hibridación de la resistencia. (CANCLINI, 1997, 44)

É evidente a preocupação latino-americana com os conceitos relacionados com a pós-modernidade e a globalização. Tal preocupação surge indubitavelmente da complexidade de uma modernização desigual e em descompasso com o centro (o que não nega necessariamente a desigualdade e o descompasso da modernização no centro também). A modernização e todos os discursos que a bordeiam modernidade, modernismos, pós-modernidade, pós-modernismos, globalização, mundialização - evidentemente formam o eixo principal dos Estudos Culturais latino-americanos contemporâneos (CANCLINI, 1999; MOREIRAS, 2001). Entretanto, são as condições da modernização latino-americana no final do século XX que vão ser focos de interesse dos teóricos latino-americanos. Nessas condições estão implicados o hibridismo, o ser periférico, as noções de caráter nacional, globalização, colonialismo e dependência. Apresentando um colóquio de cultura latino-americana em Yale em 1994, Josefina Ludmer enumera certos temas e discursos que vão aparecer nos trabalhos apresentados:

Hay una tonalidad, un conjunto de lugares comunes, que tiñe nuestra máquina "fin de siglo": modernidad y posmodernidad, nación y narración; minorías y excluidos, identidades nacionales, sexuales, raciales, culturales ("géneros" de discursos); la representación y la política; territorializaciones y desterritorializaciones, periferias, fronteras, bordes y cuerpos; el problema del lector y de la existencia misma de la literatura en la era de la información visual. Y la cultura latinoamericana en el interior de estos lugares comunes. (LUDMER, 1994, 9)

Esse conjunto de termos, além de temas específicos do colóquio, de maneira geral representa grande parte do novo paradigma das ciências sociais latino-americanas, um paradigma que tem muitos pontos em comum com a teoria pós-colonial, 
mesmo que não seja completamente coincidente. É importante notar que, especialmente a partir da década de 90, a teoria latino-americana contemporânea explicita a sua conformação às metodologias e processos próprios aos Estudos Culturais (originados nos Estudos culturais britânicos, mas com os acréscimos da teoria crítica contemporânea), começa a delinear as conexões mais diretas com a teoria pós-colonial e fixar as especificidades dos Estudos Culturais latinoamericanos:

Once this same heterogeneity and archaic latency that goes hand in hand with the aspiration to modernity is transformed into a source of Latin American exoticism in the European or North American mind, it rebounds off Latin America with the prestigious weight of those cultures (exotic, in their turn, to the eyes of Latin America), catalizing, with 'consciousness of identity', what was merely the projection of an idealized Other. In this sense, Latin American Cultural Studies, whether conducted from within the continent or abroad, assumes an ideological counter effect which is not apparent in the case of Cultural Studies pure and simple. (SEVCENKO, 1993, 148) (Uma vez que essa mesma heterogeneidade e latência arcaica que aparecem junto com a aspiração pela modernidade é transformada em uma fonte de exotismo na mente européia ou norte-americana, a América Latina é contaminada pelo peso prestigioso daquelas culturas (exóticas para os olhos latino-americanos, por sua vez), catalisando, com a "consciência de identidade" o que era meramente projeção de um Outro idealizado. Neste sentido, os Estudos Culturais Latino-Americanos, sejam eles conduzidos no continente ou no estrangeiro, assumem um contra-efeito ideológico que não é aparente no caso dos Estudos Culturais puros e simples.)

Como nos Estudos Pós-coloniais de língua inglesa, nos Estudos Culturais latino-americanos também está em jogo uma teoria da representação que necessariamente tem que levar em conta o problema da subalternidade. Inspirados 
pelo Subaltern Studies Group, uma organização de acadêmicos sul-asiáticos liderados por Ranajit Guha, alguns acadêmicos latino-americanos propuseram a formação de um grupo parecido com o Founding Statement (Discurso de fundação) do Grupo latino-americano de estudos subalternos para contrapor à historiografia tradicional da elite um estudo da cultura latino-americana que recupere as especificidades da subalternidade e corrija as distorsões estabelecidas pelas abordagens hegemônicas. Para isso é imperativa para o grupo uma revisão não só do conceito de subalternidade, como também o profundo escrutínio das concepções de nação, identidade nacional, política e cultura implicadas na história prévia do pensamento latino-americano:

To represent subalternity in Latin America, in whatever form it takes wherever it appears-nation, hacienda, work place, home, informal sector, black market- to find the blank space where it speaks as a social political subject, requires us to explore the margins of the state. (...) We must be careful, in the process of conceptualizing subalternity, not to ensnare ourselves in the problem, dominant in previous articulations of "national" liberation (...), of the national elite itself as subaltern, that is, as transcriber, translator, interpreter, editor: to avoid, in other words, the construction of postcolonial intelligentsias as "sharecroppers" in metropolitan cultural hegemony. (LATIN AMERICAN SUBALTERN STUDIES GROUP, 1993, 119) (Representar a subalternidade na América Latina, em quaisquer das formas que ela toma, em qualquer lugar que apareça - nação, hacienda, local de trabalho, casa, setor informal, mercado negro -, achar o espaço em branco onde ela fala como um sujeito social político, requer que nós exploremos as margens do estado. (...) Nós precisamos ser cuidadosos, no processo de conceituar a subalternidade, para não cairmos na armadilha do problema, dominante nas articulações prévias da liberação nacional(...), da elite nacional como subalterna, ou seja, como tradutor, transcrevente, intérprete, editor: evitar, em outras palavras, a 
construção das intelligentsias pós-coloniais como "arrendatários” na hegemonia cultural metropolitana.)

Mas, exatamente no foco de todo repensar sobre a subalternidade e suas relações com a identidade nacional e as políticas de superação do subdesenvolvimento, está a dualidade centro-periferia. Neste sentido, o debate sobre o pós-moderno serve, apesar de sua multiplicidade de "encarnações", propósitos e definições, para designar precisamente a crise de centralidade pela qual passa o Ocidente. Tal crise é uma das pedras de toque da teoria latinoamericana contemporânea, que tem buscado repensar a identidade, o hibridismo e a diferença cultural da região a partir do des-centramento pós-moderno. Curiosamente, o des-centramento vai ser muitas vezes tomado como uma inversão de valores. De repente, as margens passam a centro e o centro a margem, numa celebração catártica da diferença.

Esse viés de interpretação, corrente em diversas áreas e autores e com diversos níveis de concordância com ele, desde a abordagem literária de Carlos Rincón (1996) diferença latino-americana a partir do Boom do realismo mágico; às teorias da tradução da diferença cultural (VIEIRA, 1996); a diversos trabalhos da chilena Nelly Richard; à definição de uma cultura pós-modernista na Argentina por Santiago Colás (1994); à própria idéia de "reconversão cultural” e aos estudos sobre as cidades latino-americanas de García Canclini ou à obra sobre a pósmodernidade de Beatriz Sarlo (1994), pode ser controverso e ingenuamente otimista.

Então seria preciso tomar cuidado com a apropriação feita pelo neoliberalismo do discurso da diferença. Faz parte do próprio princípio de manutenção da hegemonia a apropriação das diferenças. A identidade cultural latino-americana é, portanto, apropriada por um sistema "multiculturalista" por sua "diferença", mas que de fato seria apenas uma "diferença" a mais. Em um certo sentido, o neoliberalismo admite, então, a diferença simplesmente porque estas formam um quadro de igualdade, um sistema de "diferenças uniformes".

That is to say, the transparency of the social has simply been transferred from the uniqueness and intelligibility of a system of equivalences to the uniqueness and intelligibility of a system of 
differences. (LACLAU e MOUFFE, 1985, 182) (O que é dizer que a transparência do social foi simplesmente transferida da unicidade e inteligibilidade de um sistema de equivalências para a unicidade e inteligibilidade de um sistema de diferenças.)

Por outro lado, cabe lembrar - algo que já foi mencionado acima- que um sistema que valoriza a diferença, estabelece uma espécie de valor positivo para sociedades culturalmente mais heterogêneas, caso da América Latina. O que por sua vez proporciona as abordagens celebratórias as quais mencionamos anteriormente. Ou seja, simultaneamente ao reconhecimento das possibilidades desse processo, há que se levar conta também os perigos da inversão de hierarquias culturais ou absolutização da diferença. Como avisa Nelly Richard,

Celebrating difference as exotic festival - a complement of otherness destined to nuance, more than subvert, the universal law- is not the same as giving the subject of this difference the right to negotiate its own conditions of discursive control, to practice its difference in the interventionist sense of rebellion and disturbance as opposed to coinciding with the predetermined meanings of the official repertory of difference. (RICHARD, 1993, 160) (Celebrar a diferença como um festival exótico - um complemento de outridade destinado a nuançar, mais que subverter, a lei universal - não é o mesmo que dar ao sujeito dessa diferença o direito de negociar suas próprias condições de controle discursivo, de praticar sua diferença no sentido intervencionista da rebelião e perturbação em contraste com a concordância com significados pré-determinados do repertório oficial da diferença.)

O hibridismo, a diferença e o reconhecimento de heterogeneidade cultural latino-americana servem como princípio de contestação - talvez excessivamente vago - da hegemonia nordocêntrica. Os mais pessimistas não hesitam em subestimar tais categorias como parte de uma ideologia da globalização que serviria a propósitos neoliberais. Uma das saídas dessa encruzilhada é mostrar-se atento às armadilhas da inversão total do esquema binário centro-periferia e 
marcar a fundamental distinção entre o autoexotismo e a consciência crítica do que constitui a identidade cultural. Como propõe Bhabha, na “epígrafe estendida” deste texto, a revisão, a reinscrição e a negociação dessas categorias, simultaneamente.

\section{REFERÊNCIAS BIBLIOGRÁFICAS}

APPADURAI, Arjun. La modernidad desbordada. Buenos Aires: Trilce/Fondo de Cultura Económica, 2001.

ASHCROFT, Bill, GRIFFITHS, Gareth, TIFFIN, Helen (eds.). The PostcolonialReader. Londres/Nova York: Routledge, 1994.

BARKER, Francis, HULME, Peter e IVERSEN, Margaret. (eds). Colonial Discourse/Post-colonial Theory. Manchester: Manchester University Press, 1994. BHABHA, Homi K.. O local da cultura. Belo Horizonte: Editora da UFMG, 1998. CANCLINI, Néstor García. Consumidores e cidadãos. Conflitos multiculturais daglobalização. Rio de Janeiro: Editora da UFRJ, 1997.

- Culturas híbridas. Estrategias para entrar y salir de la modernidad. México D.F.: Grijalbo, 1990. . La globalización imaginada. Buenos Aires: Paidós, 1999. . Imaginarios urbanos. Buenos Aires: EUDEBA, 1997.

COLÁS, Santiago. Postmodernity in Latin America: The Argentine Paradigm. Durham: Duke University Press, 1994.

DIRLIK, Arif . "The Postcolonial Aura: Third World Criticism in the Age of Global Capitalism”, Critical Inquiry 20 (1994), pp.328-356.

FEATHERSTONE, Mike. Undoing Culture. Globalization, Postmodernism and Identity. Londres/Thousand Oaks/New Delhi: Sage, 1995.

HALL, Stuart. Da diáspora. Belo Horizonte: Editora da UFMG, 2003.

LACLAU, Ernesto e MOUFFE, Chantal. Hegemony \& Socialist Strategy. Towards a Radical Democratic Politics. Londres/New York: Verso, 1985.

LATIN AMERICAN SUBALTERN STUDIES GROUP. "Founding Statement", boundary 2. 20:3 (1993), pp.110-21.

LUDMER, Josefina. "El Coloquio de Yale: máquinas de leer 'fin de siglo", La culturas de fin de siglo en América Latina. [Josefina Ludmer, ed.] Rosario:Beatriz Viterbo, 1994, pp.7-24.

MOREIRAS, Alberto. A exaustão da diferença. A política dos estudos culturais latino-americanos. Belo Horizonte: editora da UFMG, 2001.

ORTIZ, Renato. Mundialização e cultura. São Paulo: Brasiliense, 1994.

PRAKASH, Gyan. "Postcolonial Criticism and Indian Historiography", Social Text 31-32 (1992), pp.6-18.

RICHARD, Nelly. "Cultural Peripheries: Latin America and Postmodernist Decentering”, boundary 2, 20:3 (1993), pp.156-61.

. "The Latin American Problematic of Theoretical Cultural Transference: Postmodern Appropriations and Counter Appropriations",The South Atlantic Quaterly, 92:3, 1993.

. "Postmodernity and Periphery", Third Text, 2, 1987/1988, 5-12. 
RINCÓN, Carlos. "The Peripheral Center of Postmodernity: on Borges, García Márquez and Alterity",Revista Iberoamericana. Vol LXII, 176-77 (1996), pp. 16279.

ROBBINS, Bruce. "Comparative Cosmopolitanism", Social Text 31-32 (1992), pp.169-86.

ROWE, William e SCHELLING, Vivian. Memory and Modernity. Popular Culture in Latin America. Londres: Verso, 1992.

SARLO, Beatriz. Escenas de la vida posmoderna. Intelectuales, arte y videocultura en la Argentina. Buenos Aires: Ariel, 1994.

SEVCENKO, Nicolau. "Cultural Studies Questionnaire", Travesia . Journal of Latin American Cultural Studies 2:2 (1993), pp.135-49.

SHOHAT, Ella . "Notes on the 'Post-Colonial", Social Text, 31/32 (1992), pp.99113.

SPIVAK, Gayatri Chakravorty. A Critique of Postcolonial Reason. Toward a History of the Vanisinhing Present. Cambridge, MA/Londres: Harvard University Press, 1999.

.The Post-colonial Critic: Interviews, Strategies, Dialogues [ed. Sarah Harasym]. Nova York/Londres: Routledge, 1990.

VIEIRA, Else Ribeiro Pires."Nudity Versus Royal Robe: Signs in Rotation from (In)Culture to (In)Translation in Latin America", Brazil and the Discovery of America. Narrative, History, Fiction. [Bernard McGuirk, Solange Ribeiro de Oliveira, eds.] Lewiston/Queenston/Lampeter: The Edwin Mellen Press, 1996, pp. 1-15.

WEST, Cornel. "The New Cultural Politics of Difference", The Cultural Studies Reader. [Simon During, ed.] Londres/Nova York: Routledge, 1993, pp. 203-17.

YOUNG, Robert. White Mythologies. Writing History and the West. Londres/Nova York: Routledge, 1990. 